OCHA-PP-127

STUPP-98-155

hep-th/9810148

October, 1998

\title{
Supersymmetry in the AdS/CFT Correspondence
}

\author{
MAdOKA Nishimura \\ Department of Physics, Ochanomizu University \\ 2-1-1, Otsuka, Bunkyo-ku, Tokyo 112-0012, Japan \\ and \\ YOSHIAKI TANII \\ Physics Department, Faculty of Science \\ Saitama University, Urawa, Saitama 338-8570, Japan
}

\begin{abstract}
We study how local symmetry transformations of $(p, q)$ anti de Sitter supergravities in three dimensions act on fields on the two-dimensional boundary. The boundary transformation laws are shown to be the same as those of two-dimensional $(p, q)$ conformal supergravities for $p, q \leq 2$. Weyl and super Weyl transformations are generated from threedimensional general coordinate and super transformations.
\end{abstract}

*e-mail: tanii@th.phy.saitama-u.ac.jp 


\section{Introduction}

It was conjectured in ref. [1] that the string/M-theory in $(d+1)$-dimensional anti de Sitter (AdS) space times a compact space is equivalent to a $d$-dimensional conformal field theory (CFT). More precise form of this AdS/CFT correspondence was given in refs. [2], [3]. According to refs. [2], [3] the CFTs are defined on the boundary of the AdS space and the generating functional of operators $\mathcal{O}(x)$ in the boundary CFTs is given by the partition function of the string/M-theory. When the string/M-theory is represented by a low energy effective supergravity and the partition function is approximated by a stationary point of the supergravity action $S_{\text {SUGRA }}$, one obtains a relation

$$
\left\langle\exp \left(i \int d^{d} x \phi_{0}(x) \mathcal{O}(x)\right)\right\rangle_{\mathrm{CFT}}=\exp \left(i S_{\mathrm{SUGRA}}[\phi]\right)
$$

Here, $\phi_{0}$ on the left hand side are arbitrary functions defined on the $d$-dimensional boundary while $\phi$ on the right hand side are the solutions of field equations in the bulk satisfying boundary conditions $\phi=\phi_{0}$. For fields satisfying the first order field equations such as a spinor field one should impose boundary conditions on only half of the components of the fields [4], [5].

The purpose of this paper is to study how local symmetry transformations in the bulk theories act on the fields $\phi_{0}$ on the boundary. We are especially interested in how local supertransformations act on $\phi_{0}$. The fields $\phi_{0}$ on the boundary are expected to form multiplets of $d$-dimensional conformal supergravities [6], [7]. We consider three-dimensional $(p, q)$ AdS supergravities of Achúcarro and Townsend [8] in the bulk as simple examples. The AdS/CFT correspondence for three-dimensional AdS space was previously discussed from other points of view in refs. [9], [10, [1], [12].

We partially fix the gauge for the local symmetries in the bulk and obtain residual symmetries, which preserve the gauge fixing conditions. These residual symmetry transformations act on the fields non-locally in the bulk. However, they can act on the boundary fields $\phi_{0}$ locally. It is shown that the transformations of the boundary fields have a local form for $p, q \leq 2$. In particular, the supertransformations in the bulk become two-dimensional super and super Weyl transformations on the boundary, while general coordinate transformations in the bulk become general coordinate and Weyl transformations on the boundary. These transformation laws are shown 
to be exactly the same as those of two-dimensional $(p, q)$ conformal supergravities, i.e., conformal supergravities with $p$ supersymmetries of positive chirality and $q$ of negative chirality.

\section{Three-dimensional AdS supergravities}

The field content of the three-dimensional $(p, q)$ AdS supergravity [8] is a dreibein $e_{M}^{A}$, Majorana Rarita-Schwinger fields $\psi_{M}^{i}, \psi_{M}^{i^{\prime}}$ and $\mathrm{SO}(p) \times \mathrm{SO}(q)$ Chern-Simons gauge fields $A_{M}^{i j}=-A_{M}^{j i}, A_{M}^{i^{\prime} j^{\prime}}=-A_{M}^{j^{\prime} i^{\prime}}$, where $i, j, \cdots=1, \cdots, p ; i^{\prime}, j^{\prime}, \cdots=1, \cdots, q$. We denote three-dimensional world indices as $M, N, \cdots=0,1,2$ and local Lorentz indices as $A, B, \cdots=0,1,2$. Our conventions are as follows. The flat metric is $\eta_{A B}=\operatorname{diag}(-1,+1,+1)$ and the totally antisymmetric tensor $\epsilon^{A B C}$ is chosen as $\epsilon^{012}=+1.2 \times 2$ gamma matrices $\gamma_{A}$ satisfy $\left\{\gamma_{A}, \gamma_{B}\right\}=2 \eta_{A B}$. $\gamma^{\prime}$ s with multiple indices are antisymmetrized products of gamma matrices with unit strength. In particular, we have $\gamma^{A B C}=-\epsilon^{A B C}$ in three dimensions. The Dirac conjugate of a spinor $\psi$ is defined as $\bar{\psi}=\psi^{\dagger} i \gamma^{0}$. All components of gamma matrices are chosen to be real and Majorana spinors have two real components.

The Lagrangian is given by

$$
\begin{aligned}
\mathcal{L}= & \frac{1}{8 \pi G}\left[\frac{1}{2} e R+4 m^{2} e+\frac{1}{2} i \epsilon^{M N P} \bar{\psi}_{M}^{i} \mathcal{D}_{N} \psi_{P}^{i}+\frac{1}{2} i m e \bar{\psi}_{M}^{i} \gamma^{M N} \psi_{N}^{i}\right. \\
& +\frac{1}{2} i \epsilon^{M N P} \bar{\psi}_{M}^{i^{\prime}} \mathcal{D}_{N} \psi_{P}^{i^{\prime}}-\frac{1}{2} i m e \bar{\psi}_{M}^{i^{\prime}} \gamma^{M N} \psi_{N}^{i^{\prime}} \\
& -\frac{1}{4 m} \epsilon^{M N P}\left(A_{M}^{i j} \partial_{N} A_{P}^{j i}+\frac{2}{3} A_{M}^{i j} A_{N}^{j k} A_{P}^{k i}\right) \\
& \left.+\frac{1}{4 m} \epsilon^{M N P}\left(A_{M}^{i^{\prime} j^{\prime}} \partial_{N} A_{P}^{j^{\prime} i^{\prime}}+\frac{2}{3} A_{M}^{i^{\prime} j^{\prime}} A_{N}^{j^{\prime} k^{\prime}} A_{P}^{k^{\prime} i^{\prime}}\right)\right]
\end{aligned}
$$

where $m$ is a positive constant. The cosmological constant is proportional to $m^{2}$. In the following we will put the gravitational constant as $8 \pi G=1$. Our conventions for the curvature tensors are

$$
\begin{aligned}
R & =e_{A}{ }^{M} e_{B}{ }^{N} R_{M N}{ }^{A B}, \\
R_{M N}{ }^{A B} & =\partial_{M} \omega_{N}{ }^{A B}+\omega_{M}{ }^{A}{ }_{C} \omega_{N}{ }^{C B}-(M \leftrightarrow N)
\end{aligned}
$$

and the covariant derivatives are defined as

$$
\mathcal{D}_{M} \psi_{N}^{i}=\left(\partial_{M}+\frac{1}{4} \omega_{M}^{A B} \gamma_{A B}\right) \psi_{N}^{i}+A_{M}^{i j} \psi_{N}^{j}
$$




$$
\mathcal{D}_{M} \psi_{N}^{i^{\prime}}=\left(\partial_{M}+\frac{1}{4} \omega_{M}^{A B} \gamma_{A B}\right) \psi_{N}^{i^{\prime}}+A_{M}^{i^{\prime} j^{\prime}} \psi_{N}^{j^{\prime}}
$$

The covariant derivatives without $\mathrm{SO}(p) \times \mathrm{SO}(q)$ connection terms are denoted as $D_{M}$. The spin connection is given by

$$
\begin{aligned}
\omega_{M}^{A B}= & \omega_{M}^{A B}(e)+\frac{1}{4} i\left(\bar{\psi}_{M}^{i} \gamma_{A} \psi_{B}^{i}-\bar{\psi}_{M}^{i} \gamma_{B} \psi_{A}^{i}+\bar{\psi}_{A}^{i} \gamma_{M} \psi_{B}^{i}\right. \\
& \left.+\bar{\psi}_{M}^{i^{\prime}} \gamma_{A} \psi_{B}^{i^{\prime}}-\bar{\psi}_{M}^{i^{\prime}} \gamma_{B} \psi_{A}^{i^{\prime}}+\bar{\psi}_{A}^{i^{\prime}} \gamma_{M} \psi_{B}^{i^{\prime}}\right),
\end{aligned}
$$

where $\omega_{M}^{A B}(e)$ is the spin connection without torsion. If $\omega_{M}{ }^{A B}$ is treated as an independent variable in the Lagrangian (2), its field equation is solved by eq. (5).

The Lagrangian (2) is invariant under the following local transformations for arbitrary $(p, q)$ up to total derivative terms:

$$
\begin{aligned}
\delta e_{M}{ }^{A} & =\xi^{N} \partial_{N} e_{M}^{A}+\partial_{M} \xi^{N} e_{N}^{A}-\lambda^{A}{ }_{B} e_{M}{ }^{B}+\frac{1}{2} i\left(\bar{\epsilon}^{i} \gamma^{A} \psi_{M}^{i}+\bar{\epsilon}^{i^{\prime}} \gamma^{A} \psi_{M}^{i^{\prime}}\right), \\
\delta \psi_{M}^{i} & =\xi^{N} \partial_{N} \psi_{M}^{i}+\partial_{M} \xi^{N} \psi_{N}^{i}-\frac{1}{4} \lambda^{A B} \gamma_{A B} \psi_{M}^{i}-\theta^{i j} \psi_{M}^{j}+\mathcal{D}_{M} \epsilon^{i}+m \gamma_{M} \epsilon^{i}, \\
\delta \psi_{M}^{i^{\prime}} & =\xi^{N} \partial_{N} \psi_{M}^{i^{\prime}}+\partial_{M} \xi^{N} \psi_{N}^{i^{\prime}}-\frac{1}{4} \lambda^{A B} \gamma_{A B} \psi_{M}^{i^{\prime}}-\theta^{i^{\prime} j^{\prime}} \psi_{M}^{j^{\prime}}+\mathcal{D}_{M} \epsilon^{i^{\prime}}-m \gamma_{M} \epsilon^{i^{\prime}}, \\
\delta A_{M}^{i j} & =\xi^{N} \partial_{N} A_{M}^{i j}+\partial_{M} \xi^{N} A_{N}^{i j}+\mathcal{D}_{M} \theta^{i j}+2 i m \bar{\epsilon}^{[i} \psi_{M}^{j]}, \\
\delta A_{M}^{i^{\prime} j^{\prime}} & =\xi^{N} \partial_{N} A_{M}^{i^{\prime} j^{\prime}}+\partial_{M} \xi^{N} A_{N}^{i^{\prime} j^{\prime}}+\mathcal{D}_{M} \theta^{i^{\prime} j^{\prime}}-2 i m \bar{\epsilon}^{i^{\prime}} \psi_{M}^{\left.j^{\prime}\right]} .
\end{aligned}
$$

The transformation parameters $\xi^{M}(x), \lambda^{A B}(x), \theta^{i j}(x), \theta^{i^{\prime} j^{\prime}}(x)$ and $\epsilon^{i}(x), \epsilon^{i^{\prime}}(x)$ represent general coordinate, local Lorentz, $\mathrm{SO}(p) \times \mathrm{SO}(q)$ gauge and local super transformations respectively. The parameters $\epsilon^{i}, \epsilon^{i^{\prime}}$ are Majorana spinors and $\lambda^{A B}=-\lambda^{B A}, \theta^{i j}=-\theta^{j i}, \theta^{i^{\prime} j^{\prime}}=-\theta^{j^{\prime} i^{\prime}}$. The commutator algebra of these transformations closes for arbitrary $p, q$ modulo the field equations.

\section{Boundary behaviors of the fields}

It is convenient to partially fix the gauge for the local symmetries (6). We represent the three-dimensional AdS space as a region $x^{2}>0$ in $\mathbf{R}^{3}$. The boundary of the AdS space corresponds to a plane $x^{2}=0$ and a point $x^{2}=\infty$. We choose the gauge fixing condition as

$$
\begin{aligned}
& e_{M=2}^{A=2}=\frac{1}{2 m x^{2}}, \quad e_{M=2}^{a}=0, \quad e_{\mu}^{A=2}=0, \\
& \psi_{2}^{i}=0, \quad \psi_{2}^{i^{\prime}}=0, \quad A_{2}^{i j}=0, \quad A_{2}^{i^{\prime} j^{\prime}}=0,
\end{aligned}
$$


where $\mu, \nu, \cdots=0,1$ and $a, b, \cdots=0,1$ are two-dimensional world indices and local Lorentz indices respectively. The metric in this gauge has a form

$$
d x^{M} d x^{N} g_{M N}=\frac{1}{\left(2 m x^{2}\right)^{2}}\left(d x^{2} d x^{2}+d x^{\mu} d x^{\nu} \hat{g}_{\mu \nu}\right)
$$

The $\mathrm{SO}(2,2)$ invariant AdS metric corresponds to the case $\hat{g}_{\mu \nu}=\eta_{\mu \nu}$ but we consider the general $\hat{g}_{\mu \nu}$. We define $\hat{e}_{\mu}^{a}$ by $\hat{g}_{\mu \nu}=\hat{e}_{\mu}{ }^{a} \hat{e}_{\nu}{ }^{b} \eta_{a b}$.

Let us obtain asymptotic behaviors of the fields for $x^{2} \rightarrow 0$. We assume that the dreibein $e_{\mu}{ }^{a}$ behaves as $\left(x^{2}\right)^{-1}$ just as in the $\mathrm{SO}(2,2)$ invariant case. Asymptotic behaviors of other fields are determined by field equations. The field equations of the Rarita-Schwinger fields near $x^{2}=0$ are

$$
\left(x^{2} \partial_{2} \pm \frac{1}{2} \gamma_{2}\right) \psi_{\mu}=0
$$

where + is for $\psi_{\mu}=\psi_{\mu}^{i}$ and - is for $\psi_{\mu}=\psi_{\mu}^{i^{\prime}}$. The solutions behave as $\psi_{\mu \pm}^{i} \sim\left(x^{2}\right)^{\mp \frac{1}{2}}$, $\psi_{\mu \pm}^{i^{\prime}} \sim\left(x^{2}\right)^{ \pm \frac{1}{2}}$ for $x^{2} \rightarrow 0$, where the suffices \pm here denote eigenvalues of $\gamma^{2}$, i.e., chiralities in two-dimensional sense. The field equations which determine the boundary behavior of the gauge fields are

$$
\partial_{2} A_{\mu}=0
$$

for both of $A_{\mu}=A_{\mu}^{i}, A_{\mu}^{i^{\prime}}$. The solutions are independent of $x^{2}$.

According to the prescription in refs. [2], [3] one has to impose boundary conditions on the fields. As for gravity we require that the zweibein $\hat{e}_{\mu}{ }^{a}$ defined below eq. (8) approaches a given function $e_{0 \mu}{ }^{a}\left(x^{0}, x^{1}\right)$ at the boundary. Since the RaritaSchwinger fields and the Chern-Simons gauge fields have field equations which are first order in derivatives, one should impose boundary conditions on only half of their components [4], [5]. For the Rarita-Schwinger fields we impose boundary conditions on the components which become larger for $x^{2} \rightarrow 0$, i.e., $\psi_{\mu+}^{i}$ and $\psi_{\mu-}^{i^{\prime}}$. For the gauge fields all the components become independent of $x^{2}$ and one can choose either $A_{-}=e_{-}{ }^{\mu} A_{\mu}$ or $A_{+}=e_{+}{ }^{\mu} A_{\mu}$. Here, the suffices \pm denote the light-cone directions $e_{ \pm}^{\mu}=\frac{1}{\sqrt{2}}\left(e_{0}{ }^{\mu} \pm e_{1}{ }^{\mu}\right)$. We impose boundary conditions on $A_{-}^{i j}$ and $A_{+}^{i^{\prime} j^{\prime}}$. This choice is required by supersymmetry as we will see later. To summarize we impose 
boundary conditions on $e_{\mu}^{a}, \psi_{\mu+}^{i}, \psi_{\mu-}^{i^{\prime}}, A_{-}^{i j}$ and $A_{+}^{i^{\prime} j^{\prime}}$. The boundary behaviors of these fields are

$$
\begin{aligned}
& e_{\mu}{ }^{a} \rightarrow\left(2 m x^{2}\right)^{-1} e_{0 \mu}{ }^{a}, \\
& \psi_{\mu+}^{i} \rightarrow\left(2 m x^{2}\right)^{-\frac{1}{2}} \psi_{0 \mu+}^{i}, \quad \psi_{\mu-}^{i^{\prime}} \rightarrow\left(2 m x^{2}\right)^{-\frac{1}{2}} \psi_{0 \mu-}^{i^{\prime}}, \\
& A_{-}^{i j} \rightarrow 2 m x^{2} A_{0-}^{i j}, \quad A_{+}^{i^{\prime} j^{\prime}} \rightarrow 2 m x^{2} A_{0+}^{i^{\prime} j^{\prime}},
\end{aligned}
$$

where the fields with the suffix 0 are fixed functions on the boundary. Other components of the fields on the boundary are non-local functionals of the fields in eq. (11), which are obtained by solving the field equations. We also introduce notations $\psi_{0 \mu-}^{i}, \psi_{0 \mu+}^{i^{\prime}}, A_{0+}^{i}, A_{0-}^{i^{\prime}}$ defined by $\psi_{\mu-}^{i} \rightarrow\left(2 m x^{2}\right)^{\frac{1}{2}} \psi_{0 \mu-}^{i}$, etc.

\section{Local symmetries on the boundary}

Let us study how the fields on the boundary in eq. (11) transform under the residual symmetry transformations after the gauge fixing. The residual symmetries, which preserve the gauge conditions (7), are obtained by solving

$$
\begin{aligned}
& \partial_{2} \xi^{2}-\frac{1}{x^{2}} \xi^{2}=0 \\
& \partial_{2} \xi^{\mu}-\lambda^{a} \hat{e}_{a}{ }^{\mu}=0 \\
& \lambda^{2}{ }_{a} \hat{e}_{\mu}{ }^{a}-\partial_{\mu} \xi^{2}-i m x^{2}\left(\bar{\epsilon}^{i} \gamma^{2} \psi_{\mu}^{i}+\bar{\epsilon}^{i^{\prime}} \gamma^{2} \psi_{\mu}^{i^{\prime}}\right)=0 \\
& D_{2} \epsilon^{i}+m \gamma_{M=2} \epsilon^{i}+\partial_{2} \xi^{\mu} \psi_{\mu}^{i}=0 \\
& D_{2} \epsilon^{i^{\prime}}-m \gamma_{M=2} \epsilon^{i^{\prime}}+\partial_{2} \xi^{\mu} \psi_{\mu}^{i^{\prime}}=0 \\
& \partial_{2} \theta^{i j}+\partial_{2} \xi^{\mu} A_{\mu}^{i j}=0 \\
& \partial_{2} \theta^{i^{\prime} j^{\prime}}+\partial_{2} \xi^{\mu} A_{\mu}^{i^{\prime} j^{\prime}}=0 .
\end{aligned}
$$

These equations except the third one determine $x^{2}$-dependence of the transformation parameters. The third equation fixes $\lambda^{a 2}$. The general solution of eq. (12) near the boundary $x^{2}=0$ is

$$
\begin{aligned}
\xi^{2} & =-x^{2} \Lambda_{0}\left(x^{0}, x^{1}\right), \quad \xi^{\mu}=\xi_{0}^{\mu}\left(x^{0}, x^{1}\right)+\mathcal{O}\left(\left(x^{2}\right)^{2}\right) \\
\lambda^{a b} & =\lambda_{0}^{a b}\left(x^{0}, x^{1}\right)+\mathcal{O}\left(x^{2}\right), \quad \lambda^{a 2}=\mathcal{O}\left(x^{2}\right) \\
\epsilon_{ \pm}^{i} & =\left(2 m x^{2}\right)^{\mp \frac{1}{2}}\left[\epsilon_{0 \pm}^{i}\left(x^{0}, x^{1}\right)+\mathcal{O}\left(x^{2}\right)\right] \\
\epsilon_{ \pm}^{i^{\prime}} & =\left(2 m x^{2}\right)^{ \pm \frac{1}{2}}\left[\epsilon_{0 \pm}^{i^{\prime}}\left(x^{0}, x^{1}\right)+\mathcal{O}\left(x^{2}\right)\right], \\
\theta^{i j} & =\theta_{0}^{i j}\left(x^{0}, x^{1}\right)+\mathcal{O}\left(\left(x^{2}\right)^{2}\right), \quad \theta^{i^{\prime} j^{\prime}}=\theta_{0}^{i^{\prime} j^{\prime}}\left(x^{0}, x^{1}\right)+\mathcal{O}\left(\left(x^{2}\right)^{2}\right)
\end{aligned}
$$


where $\Lambda_{0}, \xi_{0}^{\mu}, \lambda_{0}^{a b}, \epsilon_{0 \pm}^{i}, \epsilon_{0 \pm}^{i^{\prime}}, \theta_{0}^{i j}$ and $\theta_{0}^{i^{\prime} j^{\prime}}$ are arbitrary functions of $x^{0}$ and $x^{1}$. Order $\mathcal{O}\left(x^{2}\right)$ and $\mathcal{O}\left(\left(x^{2}\right)^{2}\right)$ terms are non-local functionals of these functions and the fields. For instance, the order $\mathcal{O}\left(\left(x^{2}\right)^{2}\right)$ term in $\theta^{i j}$ is given by $-\int_{0}^{x^{2}} d x^{2} \partial_{2} \xi^{\mu} A_{\mu}^{i j}$. Thus, the residual symmetry transformations of the fields in the bulk of the AdS space are non-local.

However, the transformations of the fields on the boundary in eq. (11) can be local. Substituting eqs. (11), (13) into eq. (6) and taking the limit $x^{2} \rightarrow 0$ we find the bosonic transformations of the fields on the boundary as

$$
\begin{aligned}
\delta e_{0 \mu}{ }^{a} & =\xi_{0}^{\nu} \partial_{\nu} e_{0 \mu}{ }^{a}+\partial_{\mu} \xi_{0}^{\nu} e_{0 \nu}{ }^{a}+\Lambda_{0} e_{0 \mu}{ }^{a}-\lambda_{0 b}^{a} e_{0 \mu}{ }^{b}, \\
\delta \psi_{0 \mu+}^{i} & =\xi_{0}^{\nu} \partial_{\nu} \psi_{0 \mu+}^{i}+\partial_{\mu} \xi_{0}^{\nu} \psi_{0 \nu+}^{i}+\frac{1}{2} \Lambda_{0} \psi_{0 \mu+}^{i}-\frac{1}{4} \lambda_{0}^{a b} \gamma_{a b} \psi_{0 \mu+}^{i}-\theta_{0}^{i j} \psi_{0 \mu+}^{j} \\
\delta \psi_{0 \mu-}^{i^{\prime}} & =\xi_{0}^{\nu} \partial_{\nu} \psi_{0 \mu-}^{i^{\prime}}+\partial_{\mu} \xi_{0}^{\nu} \psi_{0 \nu-}^{i^{\prime}}+\frac{1}{2} \Lambda_{0} \psi_{0 \mu-}^{i^{\prime}}-\frac{1}{4} \lambda_{0}^{a b} \gamma_{a b} \psi_{0 \mu-}^{i^{\prime}}-\theta_{0}^{i^{\prime} j^{\prime}} \psi_{0 \mu-}^{j^{\prime}}, \\
\delta A_{0-}^{i j} & =\xi_{0}^{\nu} \partial_{\nu} A_{0-}^{i j}-\Lambda_{0} A_{0-}^{i j}-\lambda_{-}{ }^{-} A_{0-}^{i j}+\mathcal{D}_{0-} \theta_{0}^{i j} \\
\delta A_{0+}^{i^{\prime} j^{\prime}} & =\xi_{0}^{\nu} \partial_{\nu} A_{0+}^{i^{\prime} j^{\prime}}-\Lambda_{0} A_{0+}^{i^{\prime} j^{\prime}}-\lambda_{+}{ }^{+} A_{0+}^{i^{\prime} j^{\prime}}+\mathcal{D}_{0+} \theta_{0}^{i^{\prime} j^{\prime}}
\end{aligned}
$$

We see that the transformations with the parameters $\xi_{0}^{\mu}, \Lambda_{0}, \lambda_{0}^{a b}$ and $\theta_{0}^{i j}, \theta_{0}^{i^{\prime} j^{\prime}}$ represent general coordinate, Weyl, local Lorentz and $\mathrm{SO}(p) \times \mathrm{SO}(q)$ gauge transformations in two dimensions respectively. In particular, the general coordinate transformation in the direction $M=2$ became two-dimensional Weyl transformation. Weights of the Weyl transformation are determined by the powers of $x^{2}$ appearing in the boundary behaviors of the fields (11).

On the other hand, in the limit $x^{2} \rightarrow 0$ the fermionic transformations of the fields on the boundary in eq. (11) become

$$
\begin{aligned}
\delta e_{0 \mu}{ }^{a} & =\frac{1}{2} i\left(\bar{\epsilon}_{0+}^{i} \gamma^{a} \psi_{0 \mu+}^{i}+\bar{\epsilon}_{0-}^{i^{\prime}} \gamma^{a} \psi_{0 \mu-}^{i^{i^{\prime}}}\right), \\
\delta \psi_{0 \mu+}^{i} & =D_{0 \mu} \epsilon_{0+}^{i}+A_{0 \mu}^{i j} \epsilon_{0+}^{j}+2 m \gamma_{0 \mu} \epsilon_{0-}^{i}, \\
\delta \psi_{0 \mu-}^{i^{\prime}} & =D_{0 \mu} \epsilon_{0-}^{i^{\prime}}+A_{0 \mu}^{i^{\prime} j^{\prime}} \epsilon_{0-}^{j^{\prime}}-2 m \gamma_{0 \mu} \epsilon_{0+}^{i^{\prime}}, \\
\delta A_{0-}^{i j} & =2 i m e_{0-}{ }^{\mu}\left(\bar{\epsilon}_{0-}^{[i} \psi_{0 \mu+}^{j]}+\bar{\epsilon}_{0+}^{i} \psi_{0 \mu-}^{j]}\right)+\delta e_{0-}{ }^{\mu} A_{0 \mu}^{i j}, \\
\delta A_{0+}^{i^{\prime} j^{\prime}} & =-2 i m e_{0+}{ }^{\mu}\left(\bar{\epsilon}_{0-}^{i^{\prime}} \psi_{0 \mu+}^{\left.j^{\prime}\right]}+\bar{\epsilon}_{0+}^{i^{i^{\prime}}} \psi_{0 \mu-}^{\left.j^{\prime}\right]}\right)+\delta e_{0+}{ }^{\mu} A_{0 \mu}^{i^{\prime} j^{\prime}} .
\end{aligned}
$$

The transformation of $e_{0 \mu}{ }^{a}$ is that of the two-dimensional $(p, q)$ supergravities, i.e., supergravities with $p$ supersymmetries of positive chirality and $q$ of negative chirality (See, e.g., ref. [13].). However, the transformations of other fields have different forms 
from those of the two-dimensional supergravities. Furthermore, they contain $\psi_{0 \mu-}^{i}$, $\psi_{0 \mu+}^{i^{\prime}}, A_{+}^{i}, A_{-}^{i^{\prime}}$, which are non-local functionals of the fields in eq. (11). We shall try to rewrite these transformations in a local form by using field equations.

Using an identity

$$
\eta_{a b}=\frac{1}{2}\left(\eta_{a b}+\epsilon_{a b} \gamma^{2}\right)+\frac{1}{2} \gamma_{a} \gamma_{b}
$$

in the second terms, the transformations of the Rarita-Schwinger fields in eq. (15) can be rewritten as

$$
\begin{aligned}
& \delta \psi_{0 \mu+}^{i}=D_{0 \mu} \epsilon_{0+}^{i}+e_{0 \mu}{ }^{-} A_{0-}^{i j} \epsilon_{0+}^{j}+\gamma_{0 \mu} \eta_{0-}^{i}, \\
& \delta \psi_{0 \mu-}^{i^{\prime}}=D_{0 \mu} \epsilon_{0-}^{i^{\prime}}+e_{0 \mu}{ }^{+} A_{0+}^{i^{\prime} j^{\prime}} \epsilon_{0-}^{j^{\prime}}+\gamma_{0 \mu} \eta_{0+}^{i^{\prime}},
\end{aligned}
$$

where

$$
\begin{aligned}
& \eta_{0-}^{i}=2 m \epsilon_{0-}^{i}+\frac{1}{2} A_{0+}^{i j} \gamma^{+} \epsilon_{0+}^{j}, \\
& \eta_{0+}^{i^{\prime}}=-2 m \epsilon_{0+}^{i^{\prime}}+\frac{1}{2} A_{0-}^{i^{\prime} j^{\prime}} \gamma^{-} \epsilon_{0-}^{j^{\prime}}
\end{aligned}
$$

If we regard $\eta_{0-}^{i}, \eta_{0+}^{i^{\prime}}$ as independent transformation parameters, eq. (17) do not contain non-local functionals anymore.

As for the gauge fields we use field equations of the Rarita-Schwinger fields to eliminate $\psi_{0 \mu-}^{i}$ and $\psi_{0 \mu+}^{i^{\prime}}$. First, the first term of the transformation of $A_{0-}^{i j}$ in eq. (15) can be rewritten as

$$
i m\left(\bar{\epsilon}_{0+}^{[i} \gamma_{-} \gamma_{0}^{\nu} \psi_{0 \nu-}^{j]}+\bar{\epsilon}_{0-}^{[i} \gamma_{0}^{\nu} \gamma_{-} \psi_{0 \nu+}^{j]}\right)
$$

From the Rarita-Schwinger field equations we obtain

$$
m \gamma_{0 \mu} \gamma_{0}^{\nu} \psi_{0 \nu-}^{i}=\frac{1}{2} \gamma_{0}^{\nu} \psi_{0 \mu \nu+}^{i}-\frac{1}{4} \gamma_{0 \mu} \gamma_{0}^{\nu} A_{0}^{i j} \psi_{0 \nu+}^{j}
$$

where

$$
\begin{aligned}
& \psi_{0 \mu \nu+}^{i}=D_{0 \mu} \psi_{0 \nu+}^{i}+e_{0 \mu}{ }^{-} A_{0-}^{i j} \psi_{0 \nu+}^{j}-(\mu \leftrightarrow \nu), \\
& \psi_{0 \mu \nu-}^{i^{\prime}}=D_{0 \mu} \psi_{0 \nu-}^{i^{\prime}}+e_{0 \mu}{ }^{+} A_{0+}^{i^{\prime} j^{\prime}} \psi_{0 \nu-}^{j^{\prime}}-(\mu \leftrightarrow \nu) .
\end{aligned}
$$


$\left(\psi_{0 \mu \nu-}^{i^{\prime}}\right.$ is for later use.) Substituting eq. (20) into eq. (19) we obtain an expression for $\delta A_{0-}^{i j}$ independent of $\psi_{0 \mu_{-}}^{i}$. Similarly we obtain an expression for $\delta A_{0 \mu+}^{i^{\prime} j^{\prime}}$ independent of $\psi_{0 \mu+}^{i^{\prime}}$. Thus the transformations of the gauge fields become

$$
\begin{aligned}
\delta A_{0-}^{i j}= & \frac{1}{2} i \epsilon_{0+}^{[i} \gamma_{0}^{\nu} \psi_{0 \mu \nu+}^{j]} e_{0-}{ }^{\mu}+\frac{1}{2} i \bar{\eta}_{0-}^{[i} \gamma_{0}^{\mu} \gamma_{-} \psi_{0 \mu+}^{j]}-\frac{1}{2} i \bar{\epsilon}_{0-}^{k^{\prime}} \gamma^{-} \psi_{0 \mu-}^{k^{\prime}} e_{0-}{ }^{\mu} A_{0-}^{i j} \\
& -\frac{3}{4} i A_{0+}^{[i j} \epsilon_{0+}^{k]} \gamma^{+} \psi_{0 \mu+}^{k} e_{0-}{ }^{\mu}-\frac{3}{4} i \bar{\epsilon}_{0+}^{k} \gamma^{+} \psi_{0 \mu+}^{[i} A_{0+}^{j k]} e_{0-}{ }^{\mu}, \\
\delta A_{0+}^{i^{\prime} j^{\prime}}= & \frac{1}{2} i \epsilon_{0-}^{\left[i^{\prime}\right.} \gamma_{0}^{\nu} \psi_{0 \mu \nu-}^{\left.j^{\prime}\right]} e_{0+}{ }^{\mu}+\frac{1}{2} i \bar{\eta}_{0+}^{\left[i^{\prime}\right.} \gamma_{0}^{\mu} \gamma_{+} \psi_{0 \mu-}^{\left.j^{\prime}\right]}-\frac{1}{2} i \bar{\epsilon}_{0+}^{k} \gamma^{+} \psi_{0 \mu+}^{k} e_{0+}{ }^{\mu} A_{0+}^{i^{\prime} j^{\prime}} \\
& -\frac{3}{4} i A_{0-}^{\left[i^{\prime} j^{\prime} \bar{\epsilon}^{\prime}\right.} \bar{\epsilon}_{0-}^{\left.k^{\prime}\right]} \gamma^{-} \psi_{0 \mu-}^{k^{\prime}} e_{0+}{ }^{\mu}-\frac{3}{4} i \bar{\epsilon}_{0-}^{k^{\prime}} \gamma^{-} \psi_{0 \mu-}^{\left[i^{\prime}\right.} A_{0-}^{\left.j^{\prime} k^{\prime}\right]} e_{0+}{ }^{\mu} .
\end{aligned}
$$

The last two terms in these transformations still contain the fields $A_{0+}^{i j}$ or $A_{0-}^{i^{\prime} j^{\prime}}$, which are non-local functionals of the boundary fields. These terms vanish for $p, q \leq 2$ since three indices $i, j, k$ or $i^{\prime}, j^{\prime}, k^{\prime}$ are antisymmetrized. Therefore, we have a local form of fermionic transformations only for $p, q \leq 2$.

\section{Comparison with two-dimensional conformal supergravities}

Let us compare the above fermionic transformations of the boundary fields (15), (17), (22) obtained from the AdS/CFT correspondence with those in the twodimensional $(p, q)$ conformal supergravities for $p, q \leq 2$. We begin with the case $p=q=2$. The two-dimensional $(2,2)$ conformal supergravity [14] contains a zweibein $\tilde{e}_{\mu}{ }^{a}$, Majorana Rarita-Schwinger fields $\tilde{\psi}_{\mu}^{i}(i=1,2)$ and a real vector field $\tilde{A}_{\mu}^{i j}$. Their fermionic transformations are

$$
\begin{aligned}
& \delta \tilde{e}_{\mu}{ }^{a}=\frac{1}{2} i \overline{\tilde{\epsilon}}^{i} \gamma^{a} \tilde{\psi}_{\mu}^{i}, \quad \delta \tilde{\psi}_{\mu}^{i}=\tilde{D}_{\mu} \tilde{\epsilon}^{i}+\tilde{A}_{\mu}^{i j} \tilde{\epsilon}^{j}+\tilde{\gamma}_{\mu} \tilde{\eta}^{i}, \\
& \delta \tilde{A}_{\mu}^{i j}=\frac{1}{2} i \overline{\tilde{\epsilon}}^{i} \tilde{\gamma}_{\mu} \tilde{\gamma}^{\rho \sigma}\left(\tilde{D}_{\rho} \tilde{\psi}_{\sigma}^{j]}+\tilde{A}_{\rho}^{j] k} \tilde{\psi}_{\sigma}^{k}\right)+\frac{1}{2} i \overline{\tilde{\eta}}^{[i} \tilde{\gamma}^{\nu} \tilde{\gamma}_{\mu} \tilde{\psi}_{\nu}^{j]},
\end{aligned}
$$

where the transformation parameters $\tilde{\epsilon}^{i}$ and $\tilde{\eta}^{i}$ are Majorana spinors and represent the supertransformation and the super Weyl transformation respectively. By identifying the fields (11) with these fields as

$$
\begin{aligned}
\tilde{A}_{\mu}^{i j} & =e_{0 \mu}{ }^{-} A_{0-}^{i j}+e_{0 \mu}{ }^{+} A_{0+}^{i^{\prime} j^{\prime}}, \quad \tilde{\psi}_{\mu}^{i}=\psi_{0 \mu+}^{i}+\psi_{0 \mu-}^{i^{\prime}}, \\
\tilde{\epsilon}^{i} & =\epsilon_{0+}^{i}+\epsilon_{0-}^{i^{\prime}}, \quad \tilde{\eta}^{i}=\eta_{0-}^{i}+\eta_{0+}^{i^{\prime}}-\frac{1}{2} A_{0}^{i^{\prime} j^{\prime}} \epsilon_{0+}^{j}-\frac{1}{2} A_{0}^{i j} \epsilon_{0-}^{j^{\prime}},
\end{aligned}
$$


where $i^{\prime}=i, j^{\prime}=j$, the transformations obtained from the AdS/CFT correspondence can be shown to reproduce the fermionic transformations in eq. (23).

The fermionic transformations of the $(2,1)$ conformal supergravity can be obtained from those of the $(2,2)$ theory $(23)$ by a truncation

$$
\tilde{\psi}_{\mu-}^{2}=0, \quad \tilde{A}_{+}^{12}=0, \quad \tilde{\epsilon}_{-}^{2}=0, \quad \tilde{\eta}_{+}^{2}=\frac{1}{2} \tilde{A}^{12} \tilde{\epsilon}_{-}^{1}
$$

The transformations of the remaining fields $\tilde{e}_{\mu}{ }^{a}, \tilde{\psi}_{\mu+}^{i}, \tilde{\psi}_{\mu-}^{2}, \tilde{A}_{-}^{12}$ are exactly the same as those obtained from the AdS/CFT correspondence by an obvious identification of the fields. The $(1,1)$ theory [15] contains $\tilde{e}_{\mu}^{a}, \tilde{\psi}_{\mu}^{1}$, whose fermionic transformations are obtained from the $(2,2)$ theory by a truncation $\tilde{\psi}_{\mu}^{2}=0, \tilde{A}_{\mu}^{12}=0, \tilde{\epsilon}^{2}=0, \tilde{\eta}^{2}=0$. On the other hand, the $(2,0)$ theory [16] is obtained from the $(2,2)$ theory by a truncation $\tilde{\psi}_{\mu-}^{i}=0, \tilde{A}_{+}^{12}=0, \tilde{\epsilon}_{-}^{i}=0, \tilde{\eta}_{+}^{i}=0$. The $(1,0)$ theory [17] is obtained from the $(2,0)$ theory by further truncation $\tilde{\psi}_{\mu+}^{2}=0, \tilde{A}_{-}^{12}=0, \tilde{\epsilon}_{+}^{2}=0, \tilde{\eta}_{-}^{2}=0$. The fermionic transformations of these theories coincide with those obtained from the AdS/CFT correspondence.

Thus, for all $p, q \leq 2$ the fermionic transformations of the boundary fields are locally realized and are exactly the same as the super and the super Weyl transformations of two-dimensional $(p, q)$ conformal supergravities. For $p>2$ or $q>2$ the fermionic transformations of the gauge fields are non-local and a relation to two-dimensional conformal supergravities is not clear. We note here that the construction of the two-dimensional $(p, p)$ conformal supergravities based on the super Lie algebra $\operatorname{OSp}(2, p) \oplus \operatorname{OSp}(2, p)$ in ref. [16] also failed for $p>2$. It would be interesting to see a relation between this construction and the AdS/CFT correspondence.

\section{References}

[1] J. Maldacena, The large $N$ limit of superconformal field theories and supergravity, hep-th/9711200.

[2] S.S. Gubser, I.R. Klebanov and A.M. Polyakov, Phys. Lett. B428 (1998) 105, hep-th/9802109. 
[3] E. Witten, Anti de Sitter space and holography, hep-th/9802150.

[4] M. Henningson and K. Sfetsos, Phys. Lett. B431 (1998) 63, hep-th/9803251.

[5] G.E. Arutyunov and S.A. Frolov, On the origin of supergravity boundary terms in the AdS/CFT correspondence, hep-th/9806216.

[6] S. Ferrara, C. Frønsdal and A. Zaffaroni, On $N=8$ supergravity on $\mathrm{AdS}_{5}$ and $N=4$ superconformal Yang-Mills theory, hep-th/9802203.

[7] H. Liu and A.A. Tseytlin, $D=4$ super Yang-Mills, $D=5$ gauged supergravity and $D=4$ conformal supergravity, hep-th/9804083.

[8] A. Achúcarro and P.K. Townsend, Phys. Lett. B180 (1986) 89.

[9] J.D. Brown and M. Henneaux, Commun. Math. Phys. 104 (1986) 207.

[10] M. Bañados, K. Bautier, O. Coussaert, M. Henneaux and M. Ortiz, Phys. Rev. D58 (1998) 085020, hep-th/9805165.

[11] J. de Boer, Six-dimensional supergravity on $\mathrm{S}^{3} \times \mathrm{AdS}_{3}$ and $2 \mathrm{~d}$ conformal field theory, hep-th/9806104.

[12] A. Giveon, D. Kutasov and N. Seiberg, Comments on string theory on $\mathrm{AdS}_{3}$, hep-th/9806194.

[13] A. Salam and E. Sezgin, Supergravities in Diverse Dimensions (NorthHolland/World Scientific, 1989).

[14] L. Brink and J.H. Schwarz, Nucl. Phys. B121 (1977) 285.

[15] S. Deser and B. Zumino, Phys. Lett. B65 (1976) 369; L. Brink, P. Di Vecchia and P. Howe, Phys. Lett. B65 (1976) 471.

[16] E. Bergshoeff, E. Sezgin and H. Nishino, Phys. Lett. B166 (1986) 141.

[17] D.J. Gross, J.A. Harvey, E. Martinec and R. Rhom, Nucl. Phys. B256 (1985) 253. 